\title{
Scale-free extinction dynamics in spatially structured host-parasitoid systems
}

\author{
Timothy Killingback ${ }^{\mathrm{a}}$, Hendrik J. Blok ${ }^{\mathrm{b}}$, Michael Doebeli ${ }^{\mathrm{b}, \mathrm{c}, *}$ \\ ${ }^{a}$ Department of Mathematics, College of William and Mary, Williamsburg, VA 23187-8795, USA \\ ${ }^{\mathrm{b}}$ Department of Zoology, University of British Columbia, Vancouver, BC, Canada V6T $1 Z 4$ \\ ${ }^{\mathrm{c}}$ Department of Mathematics, University of British Columbia, Vancouver, BC, Canada V6T 1Z2
}

Received 26 July 2005; received in revised form 4 January 2006; accepted 10 January 2006

Available online 20 February 2006

\begin{abstract}
Much of the work on extinction events has focused on external perturbations of ecosystems, such as climatic change, or anthropogenic factors. Extinction, however, can also be driven by endogenous factors, such as the ecological interactions between species in an ecosystem. Here we show that endogenously driven extinction events can have a scale-free distribution in simple spatially structured host-parasitoid systems. Due to the properties of this distribution there may be many such simple ecosystems that, although not strictly permanent, persist for arbitrarily long periods of time. We identify a critical phase transition in the parameter space of the host-parasitoid systems, and explain how this is related to the scale-free nature of the extinction process. Based on these results, we conjecture that scale-free extinction processes and critical phase transitions of the type we have found may be a characteristic feature of many spatially structured, multi-species ecosystems in nature. The necessary ingredient appears to be competition between species where the locally inferior type disperses faster in space. If this condition is satisfied then the eventual outcome depends subtly on the strength of local superiority of one species versus the dispersal rate of the other.
\end{abstract}

(C) 2006 Elsevier Ltd. All rights reserved.

Keywords: Extinction dynamics; Spatially structured ecosystems; Scale-free processes; Predator-prey dynamics; Biodiversity

\section{Introduction}

Understanding the ecological processes responsible for the persistence and extinction of biological populations is a fundamental goal of ecological theory (Diamond, 1984; Lawton and May, 1995; Mayr, 1985; Simberloff, 1984; Wilson, 1988, 1992). The simplest organizing principle for studying the question of persistence in multi-species communities is to divide ecosystems into two classes: those that are permanent, in the sense that all species coexist indefinitely, and those that are not, so that some species are driven to extinction in a finite time. If one is interested in the causes of persistence versus extinction then it is important to be able to classify these systems correctly.

\footnotetext{
*Corresponding author. Department of Zoology, University of British Columbia, 6270 University Boulevard, Vancouver, BC, Canada V6T $1 Z 4$. Tel.: + 16048223326 .

E-mail address: doebeli@zoology.ubc.ca (M. Doebeli).
}

Thus, one would like to understand the statistical distribution of extinction events in a given class of ecosystems. For example, in such an approach we would be interested in determining the distribution of times until the first species in a community becomes extinct, for a large number of communities in a given class. If the distribution of extinction times is such that many communities that are not strictly permanent can survive for long periods of time, then the relevant notion of ecological persistence must be broadened to include many non-permanent ecosystems. Therefore, the fundamental issue of understanding persistence in multi-species communities depends critically on the particular form of the distribution of extinction times.

Multi-species, host-parasitoid ecosystems are well suited to the theoretical investigation of this issue for a number of reasons. First, insect parasitoids are extraordinarily abundant, comprising approximately $10 \%$ of all metazoan species (Godfray, 1994) so any findings pertain to a significant fraction of all animal species. Second, the 
relatively uncomplicated nature of the life cycle of parasitoids means that reasonably simple models (Hassell, 1978, 2000) can describe host-parasitoid population dynamics. Third, many aspects of host-parasitoid systems (such as multi-species interactions (Hassell, 1978, 2000) have been studied theoretically. Finally, the ability to study host-parasitoid interactions in laboratory microcosms means that theoretical results on extinction dynamics in such systems may possibly be tested experimentally.

Without spatial structure these models are well understood. Indeed, many of them can be solved analytically. Incorporating spatial structure serves a number of purposes. Firstly, it is realistic - few natural ecosystems are so small that space can be said to be unimportant. Secondly, it has been previously explored (Comins et al., 1992; Comins and Hassell, 1996; Hassell, 2000; Hassell et al., 1991, 1994) and shown to introduce novel and interesting effects. Finally, spatial separation enables the "rescue effect" (recolonization after local extinction), of particular importance in systems like the one we will be investigating, where the local dynamics are unstable. Therefore, spatially structured host-parasitoid models provide natural model ecosystems with which to investigate extinction processes.

Here we study extinction dynamics in a class of simple multi-species, host-parasitoid ecosystems. We use models that can exhibit permanence of all species in the community as well as extinction of one or more species. We show that these regimes are separated by a critical phase transition, and that, as a consequence, the extinction dynamics in these systems has a distinctive statistical distribution - it is a scale-free process governed by a power law. We also show that species may persist despite the presence of superior competitors by taking advantage of space through dispersal.

\section{Model}

A convenient simple system for studying community extinction dynamics consists of two parasitoid species attacking a common host species in a spatially structured habitat that is divided into a large number of discrete patches (Comins and Hassell, 1996; Hassell, 2000; Hassell et al., 1994). In a homogeneous environment, the population dynamics of this host-parasitoid system may be described by the following system of difference equations (Comins and Hassell, 1996; Hassell et al., 1994):

$N_{t+1}=\lambda N_{t} \exp \left(-R_{t}\right)$

$P_{t+1}=N_{t}\left[1-\exp \left(-R_{t}\right)\right]\left(P_{t} / R_{t}\right)$,

$Q_{t+1}=N_{t}\left[1-\exp \left(-R_{t}\right)\right]\left(\beta Q_{t} / R_{t}\right)$.

In these equations, $N_{t}, P_{t}$ and $Q_{t}$ denote the population size, in generation $t$, of the host and the two parasitoid species, respectively. $\lambda$ is the growth rate of the host in the absence of any parasitoids. $\beta=a_{Q} c_{Q} / a_{P} c_{P}$ is the relative superiority of parasite $Q$. (The $a$-values are the per capita attack rates for each parasitoid species and the $c$-values are the average numbers of adult female parasitoids of each species emerging from each parasitized host. These four $a$ and $c$ parameters are over-specified and can be replaced with the single parameter $\beta$.) Finally, $R_{t}=P_{t}+\beta Q_{t}$ is the effective parasite population level. Without loss of generality we can assume $\beta>1$; accordingly, the parasitoid with density $P_{t}$ is called the inferior-type parasitoid, while the parasitoid with density $Q_{t}$ is called the superior-type parasitoid, because the ratio $Q_{t} / P_{t} \propto \beta^{t}$ increases with time. In formulating this model it is assumed that the two types of parasitoids search independently and randomly, and hence the population dynamics can be described in classical Nicholson-Bailey form (Hassell, 2000). It is also assumed that the two parasitoid species search simultaneously for hosts and that superparasitism by either parasitoid species does not occur. The dynamics of this spatially unstructured model is such that both parasitoid species are quickly driven to extinction (Comins and Hassell, 1996; Hassell et al., 1994).

In a spatially structured habitat, consisting of a large number of patches, which we represent by the cells of a $32 \times 32$ square lattice (larger lattices give qualitatively similar results but are computationally prohibitive), the dynamics of this host-parasitoid system can be regarded as having two stages. In the first the host and parasitoid populations in each patch reproduce according to the system of equations given above. In the second phase fractions $\mu_{N}, \mu_{P}$ and $\mu_{Q}$, respectively, of the host and parasitoid populations in each patch disperse and are distributed equally among the eight nearest neighbours of that patch in the square lattice (Comins et al., 1992; Comins and Hassell, 1996; Hassell, 2000; Hassell et al., 1991, 1994).

\section{Results}

The fundamental theoretical issue concerning the extinction dynamics is to determine the distribution of times to extinction of at least one member of the community, for a large ensemble of host-parasitoid systems of the type described above, with regularly sampled parameters. We found that the extinction dynamics depended most sensitively on just two parameters: the relative superiority $\beta$, and the quotient of the two parasitoid dispersal fractions $\mu=\mu_{P} / \mu_{Q}$. Thus, we worked with fixed host properties $\lambda=2$ and $\mu_{N}=1 / 2$, and varied $\beta, \mu_{P}$ and $\mu_{Q}$ to sample parameter space. For each set of parameters we simulated the host-parasitoid system to determine whether any species go extinct. Local populations were set to zero if they fell below a specified threshold $\varepsilon$ (taken to be 10 orders of magnitude below the (unstable) equilibrium density of the non-spatial model), and a species was deemed to have gone extinct if it had zero population density in every patch. We determined the number of host-parasitoid systems $E(t)$ in which all species survive for at least time $t$ from these simulations in the following way. We constructed a regular sampling of the parameter space of 
$\left(\beta, \mu_{P}, \mu_{Q}\right)$ values, with each point in this sampling representing a spatial host-parasitoid system. For each of these host-parasitoid systems we determined by simulation the time until the first species went extinct (this time was taken to be infinity if all species were permanent), and from this determined the distribution $E(t)$. Thus, if spatial host-parasitoid systems are picked at random then the corresponding extinction times will be distributed according to $E(t)$. The distribution of $E(t)$ against $t$ is shown in Fig. 1. We found that $E(t)$ is described well by a power law $E(t) \propto t^{-\eta}$ for large $t$, with an exponent $\eta \approx 2.2$. Extensive simulations show that the power-law behaviour of the distribution $E(t)$ is robust to changes in the parameters $\lambda$ and $\mu_{N}$.

Power-law distributions are interesting because they are scale-free, meaning that they look the same on all scales of the property being measured. For instance, in our current study we could predict exactly the same shape for the distribution $E(t)$ whether we studied time-scales of decades or millennia. (This follows directly from $E(u) \propto u^{-\eta} \propto t^{-\eta}$, where $u=t / s$ is just $t$ measured in different units.) Therefore, there is no characteristic time-scale governing extinction dynamics, and extinctions occur on all time scales, with some communities losing species rapidly, while others maintain all species for long periods of time. Further, since the exponent $\eta<3$ the variance of the distribution is unbounded: the second moment of the distribution is $\int E(t) t^{2} \mathrm{~d} t \propto \int t^{2-\eta} \mathrm{d} t$, which is infinite for $\eta<3$. One important consequence of this is that there exist many non-permanent host-parasitoid systems that persist for arbitrary long periods of time. From a practical ecological point of view such long-lived ecosystems are essentially permanent.

Two general types of dynamic regimes can be distinguished in our models: for some parameter values all

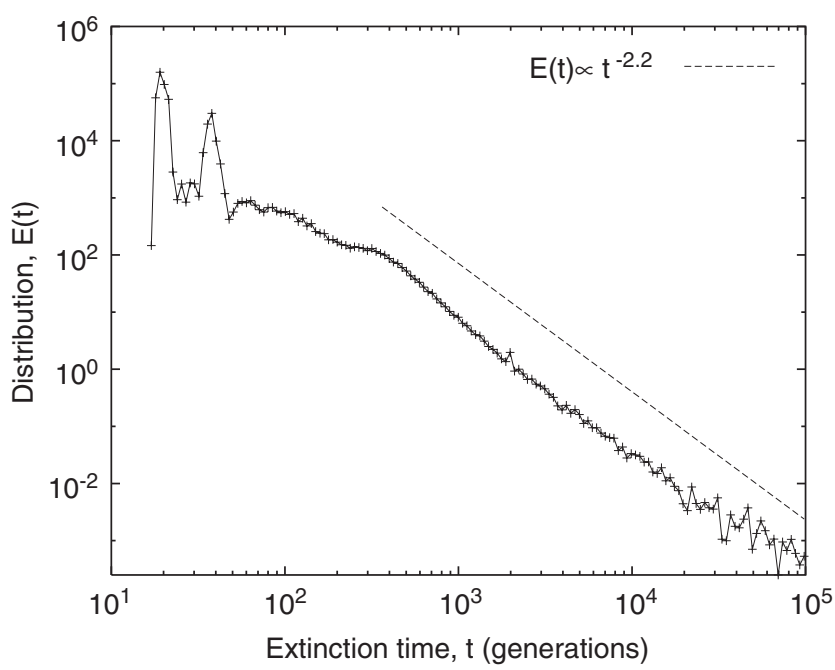

Fig. 1. Distribution of extinctions in a regular sampling of parameter space, showing a power-law tail $E(t) \propto t^{-\eta}$ with $\eta \approx 2.2$. [Sample size, $N=691,805$ extinctions; $32 \times 32$ lattice; parameter space: dispersal rates $\mu_{P}, \mu_{Q}=0.01,0.02, \ldots, 0.99$, relative superiority of one parasite $\beta=1.01$, $1.03, \ldots, 3.99$.

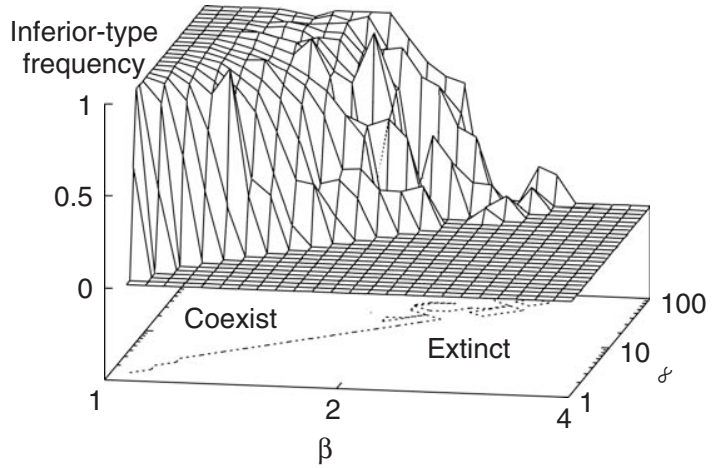

(a)
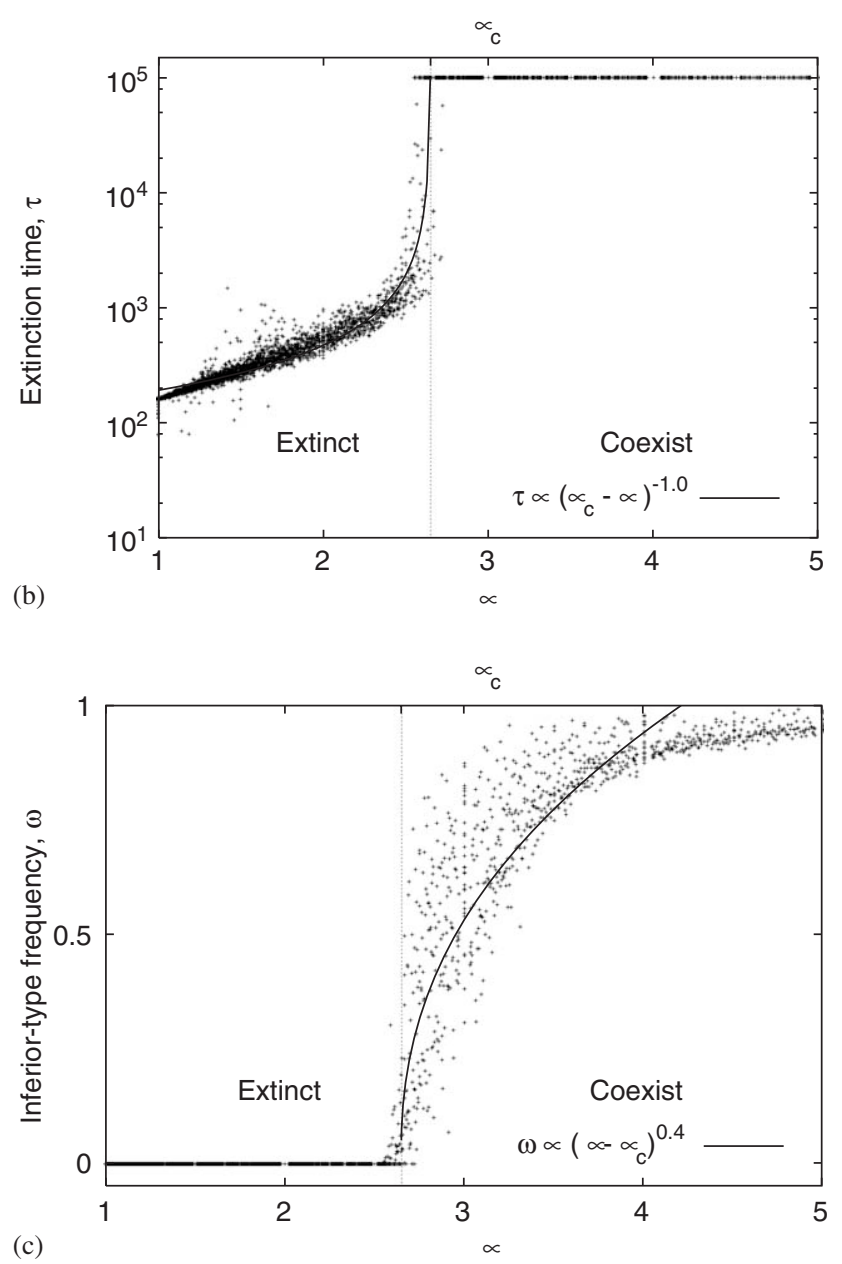

Fig. 2. The inferior-type can coexist with the superior-type even when it is much less efficient if it has a high migration rate. (a) The frequency $\omega$ of the inferior-type after 100,000 generations demonstrates the two phases: coexisting and extinct. The boundary between the regimes is shown on the floor of the plot. [Parameter details: relative dispersal rate $\mu=\mu_{P} / \mu_{O} ; \mu_{P}$ was fixed at 0.99 (results are qualitatively the same for other values of $\mu_{P}$ ); averaged over four replicates.] Fixing the relative superiority of one parasite $\beta$ and varying the relative dispersal rate $\mu$ of the other demonstrates that power laws characterize the transition on both sides. (b) Time (generations) until the inferior-type goes extinct: $\tau \propto\left(\mu_{c}-\mu\right)^{-1.0}$. (c) Frequency of the inferior-type after 100,000 generations: $\omega \propto\left(\mu-\mu_{c}\right)^{0.4}$. [Subset of Fig. 1 dataset with $\beta=1.15$ in panels (b) and (c).] 
species coexist indefinitely while for others one or more of the species goes extinct (Fig. 2a). In particular, if the relative superiority $\beta$ of one parasite is fixed while the dispersal rate of the other $\mu=\mu_{P} / \mu_{Q}$ is varied, our models exhibit a phase transition: extinction occurs for low values of $\mu$, while all species coexist indefinitely as $\mu$ is increased beyond a critical point $\mu_{c}$ (Fig. 2b). In physics, phase transitions are called critical if the system behaviour near the critical point is governed by power laws due to system fluctuations that can grow to be of any size and produce events on all scales. In our models the continuous nature of the divergence of the time to extinction of the inferior-type parasitoid, $\tau$, at the critical point $\mu_{c}$, as shown in Fig. 2b, demonstrates that there is a critical phase transition at $\mu_{c}$ (Goldenfeld, 1992). More specifically, the divergence in extinction time near the critical point is itself governed by a power law, $\tau \propto\left(\mu_{c}-\mu\right)^{-\kappa}$, which is characteristic of a critical phase transition (Goldenfeld, 1992). Other natural statistical measures of the extinction dynamics also have power-law behaviour. An example is the frequency $\omega$ of the inferior-type parasitoid as a function of $\mu$. We find that $\omega$ declines continuously as $\mu$ is decreased to $\mu_{c}$, and that a power law describes the divergence: $\omega \propto\left(\mu-\mu_{c}\right)^{\sigma}$ (Fig. 2c). The behavior of $\tau$ and $\omega$ demonstrates that power laws hold on both sides of the phase transition.

The existence of a critical phase transition in our model provides an explanation for the power laws that govern the distribution of extinction times $E(t)$. When calculating $E(t)$ from a large ensemble of host-parasitoid systems, those corresponding to parameter sets near the critical phase transition will have extinction dynamics governed by a scale-free process described by power laws. Parameter sets far from the critical phase transition correspond to ecosystems in which either both parasitoid species coexist indefinitely or one parasitoid species goes extinct rapidly. Ecosystems in the former category do not contribute to the extinction statistics, while those in the latter category contribute a peak in $E(t)$ at short times (see Fig. 1). Thus, overall the dominant contribution to $E(t)$ comes from host-parasitoid systems corresponding to parameter sets near the critical phase transition, which generates the power-law behaviour.

\section{Variants}

It is important to note that the phenomena we have reported here are not restricted to one particular system. We have also studied in detail the extinction dynamics of a number of other multi-species spatial ecosystems: the first consists of a single parasitoid species attacking two host species (Comins and Hassell, 1996; Hassell, 2000; Hassell et al., 1994). This example of apparent competition between host species also exhibits a scale-free distribution of extinction times and a critical phase transition between extinction and coexistence regimes (see Fig. 3).

In the second alternative model we replaced the host in the original model by completely random values (updated
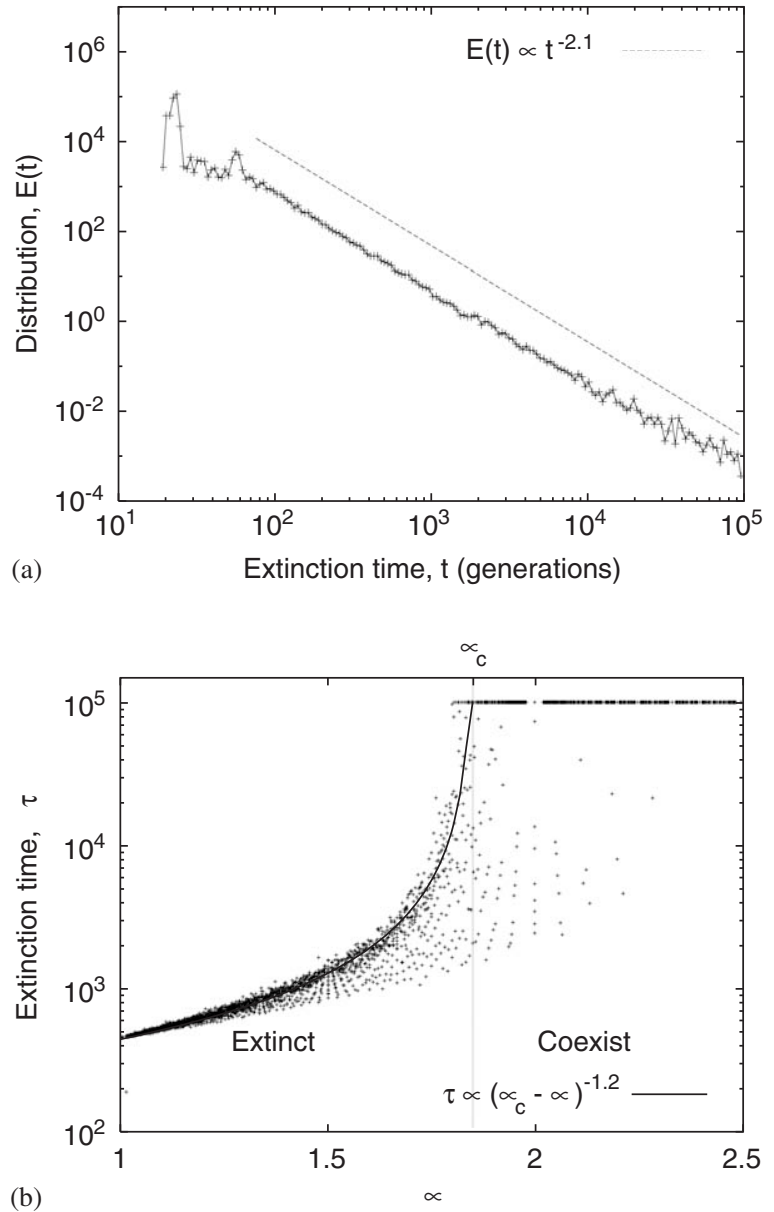

Fig. 3. Another variant, the 2-host Nicholson-Bailey model, also exhibits (a) power law extinction times: $E(t) \propto t^{-2.1}$; and (b) a critical phase transition between extinction and coexistence regimes: $\tau \propto\left(\mu_{c}-\mu\right)^{-1.2}$ (at $\beta=1.05)$. Compare with Figs. 1 and 2 (b), respectively. $(32 \times 32$ lattice. Parameter space: $\mu_{P}, \mu_{Q}=0.01,0.02, \ldots, 0.99, \mu=\mu_{P} / \mu_{Q}$.)

every generation) in order to demonstrate the phase transition is not dependent on the particulars of the host-parasite interaction. Even in this simpler two-species system with noise, the results are qualitatively similar to the case where the host dynamics are strictly determined by the host-parasite interactions (see Fig. 4a).

In yet another variation we replaced the two-dimensional lattice by a one-dimensional chain of connected sites, representing a ring-ecology as around a lakeshore. We again observed a phase transition between coexistence and extinction as shown in Fig. 4b. The critical exponents found in this one-dimensional situation were different from those found in the two-dimensional cases (for example, the extinction-time power law had a different exponent, $\left.E(t) \propto t^{-1.7}\right)$, indicating that the nature of the critical point depends on the spatial structure of the system, as is expected for phase transitions (Goldenfeld, 1992). Thus, although the exact rate at which $E(t)$ decreases with time depends on the particular system (including the spatial dimension), the power-law distribution $E(t) \propto t^{-\eta}$ holds for all systems we have studied. The power-law distribution of $E(t)$ is the feature of key ecological significance since it 

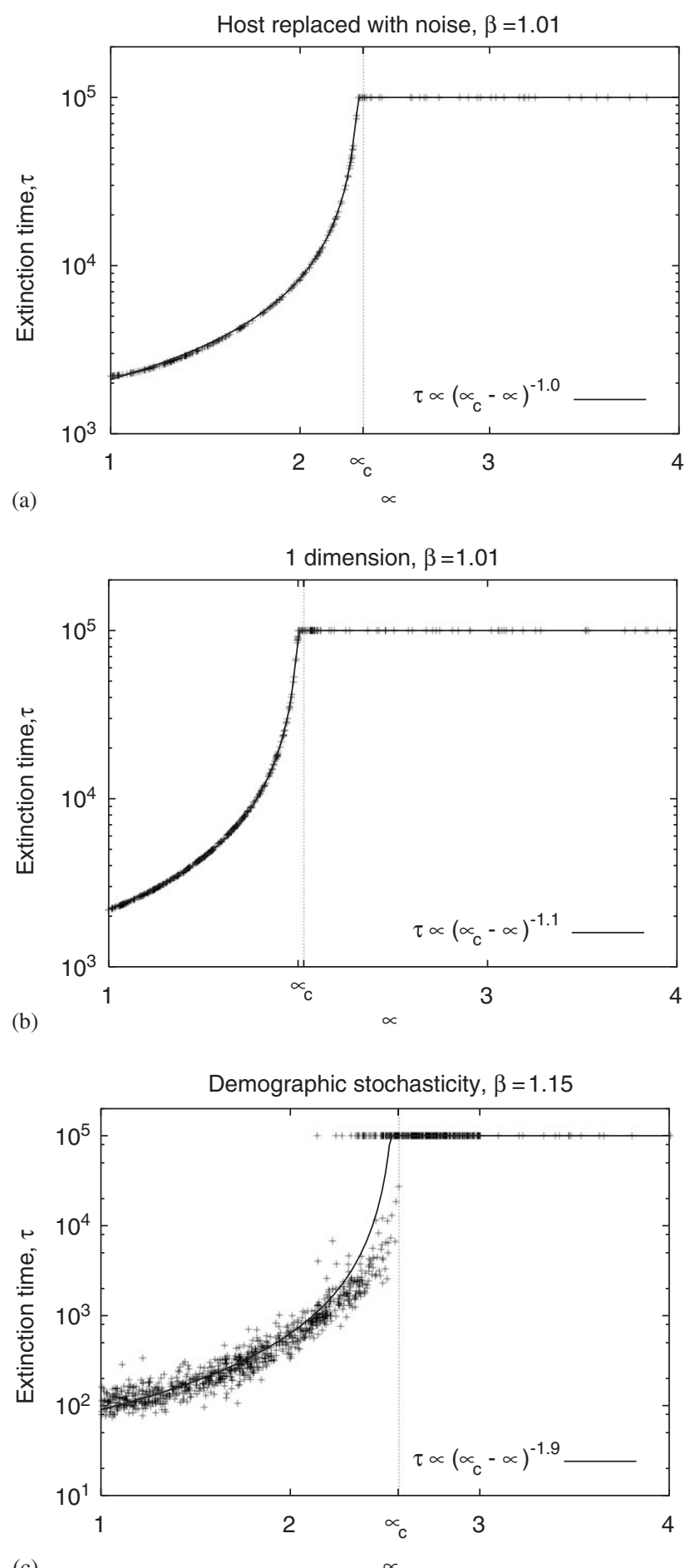

(c)

$\mu$

Fig. 4. Three variants of our model exhibit the same pattern of a diverging extinction time leading to permanence as the inferior-type's migration rate $\mu$ increases as seen in Figs. 2(b) and 3(b). Here the host has been replaced by a random variable in panel (a); the spatial grid is constrained to one dimension [1024 $\times 1$ lattice] in panel (b); and demographic stochasticity has been introduced in panel (c). See the text for more details. Although the pattern is consistent the details, such as the critical points $\mu_{c}$ and the power-law exponents, are not universal. [Parameter space: $\mu_{P}=0.99$ in all simulations, $\mu=\mu_{P} / \mu_{Q}$.] implies that there exist many non-permanent ecosystems in which all species persist for arbitrarily long periods of time.

Finally, we modified our original model to study the effect of demographic stochasticity. We were able to imitate an individual-based simulation by replacing the bulk population dynamics with stochastic deviates sampled from appropriate distributions. The values computed by the deterministic equations were interpreted as averages of distributions that were then sampled to produce actual population levels. Both the birth-death and dispersal processes incorporated demographic stochasticity in this way, but with different underlying distributions: births and deaths were assumed to obey a Poisson process characterized by an average net birth rate (per generation) given by the Nicholson-Bailey equations shown above; dispersals followed a binomial distribution where the fractions $\mu_{N}, \mu_{P}$ and $\mu_{Q}$ determined the average number of individuals to disperse. Even if these assumptions do not exactly mimic the intended individual-based dynamics, they capture two important features: they introduce a stochastic effect and the stochasticity grows with small populations. (Our original model represents the limiting case of infinitely large populations so that fluctuations become infinitesimal.) Even in the limit of extremely small populations, this model also exhibited the same phase transition as seen in the original model and the other variants (see Fig. 4c).

\section{Discussion}

We have investigated the dynamics of endogenously driven extinctions in a simple class of spatially structured, multi-species host-parasitoid systems. Our models for spatial communities can exhibit permanence of all species in the community as well as extinction of one or more species. Permanence is in itself an interesting model outcome, because not only does it imply that inherently unstable host-parasitoid interactions can persist in spatially structured populations (as shown by Hassell et al., 1994), but it also implies that a parasitoid (or host) type that is locally always inferior to another type can nevertheless persist if it can take advantage of space through dispersal. Nevertheless, extinctions occur for large regions of parameter space, and here we have shown that the two regimes of permanence and extinction are separated by a critical phase transition. As a consequence, extinction is a scale-free process in these systems, and power laws govern the distribution of extinction times.

It is interesting to note that a number of distinct mechanisms can in principle lead to scale-free distributions. The mechanism that is relevant here is that near a critical phase transition correlation scales become infinite and the system becomes scale-free (Goldenfeld, 1992). Other mechanisms, which can also result in scale-free distributions in various systems, include: self-organized criticality (Bak et al. 1987), in which systems self-organize into a critical state which is scale-free; "rich-get richer" mechanisms, in which the probability of an individual choosing a 
particular option increases with the number of individuals that have already chosen it - this type of mechanism can lead to scale-free distributions in, for example, citation statistics, city sizes, and degree distributions of networks (see, for example, Newman, 2003); and random processes, such as Brownian motion (see, for example, Hastings and Sugihara, 1993). Our identification of a critical phase transition in the spatially structured ecological models we have studied makes it clear that this is the origin of the scale-free extinction dynamics that we have found. We have no reason to believe that any other mechanism (such as any of those mentioned above) is involved.

Consequently, there exist many ecosystems of this type that, although not strictly permanent, persist for long periods of time. The existence of long-lived, non-permanent ecosystems that results from power-law extinction dynamics can be contrasted with the case of extinction statistics having an exponential distribution, which would result if each species had a constant probability per unit time of going extinct. Such a distribution is not scale-free, but rather has a "half-life" which characterizes the timescale on which extinctions occur, and there would be essentially no ecosystems that would persist for significantly longer than the characteristic "half-life".

We have found scale-free extinction dynamics, and the associated critical phase transition, in different kinds of simple, spatially structured, multi-species host-parasitoid systems. Competition between different species is a key feature of these ecosystems and occurs either because two parasitoid species are competing directly for the host species, or because the two host species are in apparent competition due to the common parasitoid species attacking both. We have shown that the critical phase transition is preserved in many variants of the model. Thus, we conjecture that the phenomena we have found here are characteristic of a large class of spatially structured ecosystems, in which there are competitive interactions between different species, and populations in neighbouring habitat patches are coupled by dispersal. Establishing the veracity of this conjecture would seem to be an important topic for future research.

Previous approaches to understanding spatial mechanisms for species coexistence (Comins et al., 1992; Comins and Hassell, 1996; Hassell et al., 1991, 1994; King and Hastings, 2003) have focused on the role of spatial structure in promoting species permanence. The existence of scalefree extinction dynamics implies that the conventional notion that persistence of a community is equivalent to permanence must be refined to allow for the existence of many non-permanent ecosystems that persist for long periods of time. Thus, scale-free extinction dynamics represents a novel and potentially important mechanism through which spatial structure leads to species coexistence.

Finally, it is interesting to note that near a critical phase transition, system often exhibit complex transient dynamics that lasts for long periods of time before relaxing to some simple asymptotic state (Killingback and Doebeli, 1998).
Such supertransients are known to occur in the time-series of various spatially structured ecological models (Hastings, 2004; Hastings and Higgins, 1994). These supertransients result from the existence of a chaotic saddle in the ecological model (Hastings, 2004). Our results suggest that a new type of supertransient may occur in certain spatially structured ecological models as a consequence of a critical phase transition in the model. If this is the case then this type of critical phase transition may also have important implications for the nature of population dynamics.

\section{Acknowledgements}

MD was supported by the James S. McDonnell Foundation (USA) and by the Natural Sciences and Engineering Research Council, Canada.

\section{References}

Bak, P., Tang, C., Wiesenfeld, K., 1987. Self-organized criticality: an explanation of $1 / f$ noise. Phys. Rev. Lett. 59, 381-384.

Comins, H.N., Hassell, M.P., 1996. Persistence of multispecies host-parasitoid interactions in spatially distributed models with local dispersal. J. Theor. Biol. 183, 19-28.

Comins, H.N., Hassell, M.P., May, R.M., 1992. The spatial dynamics of host-parasitoid systems. J. Anim. Ecol. 61, 735-748.

Diamond, J.M., 1984. "Normal" extinctions of isolated populations. In: Nitecki, M.H. (Ed.), Extinctions. Chicago University Press, Chicago, pp. 191-246.

Godfray, H.C.J., 1994. Parasitoids: Behavioral and Evolutionary Ecology. Princeton University Press, Princeton, NJ.

Goldenfeld, N., 1992. Lectures on Phase Transitions and the Renormalization Group. Addison-Wesley, Reading, MA.

Hassell, M.P., 1978. The Dynamics of Arthropod Predator-Prey Systems. Princeton University Press, Princeton, NJ.

Hassell, M.P., 2000. The Spatial and Temporal Dynamics of Host-Parasitoid Interactions. Oxford University Press, Oxford.

Hassell, M.P., Comins, H.N., May, R.M., 1991. Spatial structure and chaos in insect population dynamics. Nature 353, 255-258.

Hassell, M.P., Comins, H.N., May, R.M., 1994. Species coexistence and self-organizing spatial dynamics. Nature 370, 290-292.

Hastings, A., 2004. Transients: the key to long-term ecological understanding? TREE 19, 39-45.

Hastings, A., Higgins, K., 1994. Persistence of transients in spatially structured ecological models. Science 263, 1133-1136.

Hastings, H., Sugihara, G., 1993. Fractals: A Users Guide for the Natural Sciences. Oxford University Press, Oxford.

Killingback, T., Doebeli, M., 1998. Self-organized criticality in spatial evolutionary game theory. J. Theor. Biol. 191, 335-340.

King, A.A., Hastings, A., 2003. Spatial mechanisms for coexistence of species sharing a common natural enemy. Theor. Pop. Biol. 64, 431-438.

Lawton, J.H., May, R.M. (Eds.), 1995. Extinction Rates. Oxford University Press, Oxford.

Mayr, E.E., 1985. The Growth in Biological Thought. Belknap Press, Cambridge, MA.

Newman, M., 2003. The structure and function of complex networks. SIAM Rev. 45, 167-256.

Simberloff, D.S., 1984. Mass extinction and the destruction of moist tropical forests. Zh. Obshch. Biol. 45, 767-778.

Wilson, E.O., 1988. Biodiversity. National Academy Press, Washington, DC.

Wilson, E.O., 1992. The Diversity of Life. Harvard University Press, Cambridge, MA. 